\title{
A randomized controlled trial of Explore Transplant at Home to improve transplant knowledge and decision-making for CKD 3-5 patients at Kaiser Permanente Southern California
}

Amy D. Waterman ${ }^{1,2^{*}}$ D, Crystal Anderson ${ }^{1,2}$, Angelika Alem³ ${ }^{3}$, John D. Peipert ${ }^{1,2,4}$, Jennifer L. Beaumont ${ }^{2}$, Shayna L. Henry ${ }^{3}$, Bhanuja Dub ${ }^{3}$, Lizeth Ambriz ${ }^{1,2}$, Neha Bijjala ${ }^{1,2}$, Amanda Faye Lipsey $^{2}$ and Brian Mittman ${ }^{3}$

\begin{abstract}
Background: Five-year survival on dialysis is only $40 \%$, compared to $74 \%$ with a deceased donor kidney transplant (DDKT) and 87\% with a living donor kidney transplant (LDKT). An American Society of Transplantation (AST) Consensus Conference recommended that patients with chronic kidney disease (CKD) Stages 3-5 have the opportunity to learn about and decide which treatment option is right for them, particularly about LDKT. However, early education about LDKT and DDKT outside of transplant centers is inconsistent and often poor, with patients in CKD 3 and 4 and ethnic/ racial minorities even less likely to receive it. A new randomized control trial $(\mathrm{RCT})$, in partnership with Kaiser Permanente Southern California (KPSC), will assess knowledge gaps and the effectiveness of a supplementary video-guided, print and technology-based education intervention for English- and Spanish-speaking patients in CKD Stages 3, 4, and 5 to increase LDKT knowledge and decision-making. To date, no published LDKT educational interventions have studied such a large and diverse CKD population.
\end{abstract}

Methods: In this RCT, 1200 English and Spanish-speaking CKD Stage 3-5 patients will be randomly assigned to one of two education conditions: ET@Home or KPSC standard of care education. Randomization will be stratified by CKD stage and primary language spoken. Those in the ET@Home condition will receive brochures, postcards, DVDs, and text messages delivering educational content in modules over a six-month period. Baseline data collection will measure demographics, transplant derailers, and the amount of previous CKD and transplant education they have received. Changes in CKD and transplant knowledge, ability to make an informed decision about transplant, and self-efficacy to pursue LDKT will be captured with surveys administered at baseline and at six months.

Discussion: At the conclusion of the study, investigators will understand key knowledge gaps for patients along the CKD continuum and between patients who speak different languages and have assessed the effectiveness of both English- and Spanish-language supplementary education in increasing KPSC patients' knowledge about the opportunities for and risks and benefits of LDKT. We hope this program will reduce disparities in access to transplant.

\footnotetext{
* Correspondence: awaterman@mednet.ucla.edu

'Division of Nephrology, David Geffen School of Medicine, University of

California Los Angeles, Los Angeles, California, USA

${ }^{2}$ Terasaki Research Institute, Terasaki Research Institute, 1018 Westwood Blvd,

Los Angeles, CA 90024, USA

Full list of author information is available at the end of the article
}

C The Author(s). 2019 Open Access This article is distributed under the terms of the Creative Commons Attribution 4.0 International License (http://creativecommons.org/licenses/by/4.0/), which permits unrestricted use, distribution, and reproduction in any medium, provided you give appropriate credit to the original author(s) and the source, provide a link to the Creative Commons license, and indicate if changes were made. The Creative Commons Public Domain Dedication waiver (http://creativecommons.org/publicdomain/zero/1.0/) applies to the data made available in this article, unless otherwise stated. 
(Continued from previous page)

Trial Registration: ClinicalTrials.gov Identifier: NCT03389932; date registered: 12/26/2017.

Keywords: Kidney transplantation, Living donor, Racial disparities, African-Americans, Hispanics, Patient education, Health knowledge/attitudes, Transtheoretical model

\section{Background}

There are 30 million Americans with chronic kidney disease (CKD) and at risk for kidney failure, and 660,000 patients with end-stage renal disease (ESRD). To sustain life, ESRD patients, 31\% of whom are Black, 15\% Hispanic, and 5\% Asian, must either receive daily or weekly dialysis treatments or have a kidney transplant. The five-year survival rate for patients on dialysis is only $40 \%$, compared to $74 \%$ for patients who receive a deceased donor kidney transplant (DDKT) and $87 \%$ for patients who receive a living donor kidney transplant (LDKT) [1]. Over 95,000 individuals are awaiting a DDKT in the United States, with more being added daily (based on OPTN data as of 06/08/2018).

Patients who receive kidney transplants live 5-15 years longer than if they remained on dialysis and have a better health-related quality of life (HRQOL), including a greater likelihood of being in the workforce during their critical earning years [2]. While LDKT is the medically optimal and most cost-effective renal replacement therapy (RRT) for patients with ESRD, LDKT rates have declined by $17 \%$ from 2004 to 2014. In 2014, in the U.S., 17,106 patients received a transplant (5536 LDKTs) while 8021 patients died or became too ill to remain on the list; $70 \%$ who died were racial/ethnic minorities. Of the 2281 transplants performed in California in 2017, only 604 were from living donors (OPTN data as of 06/08/2018).

Providing comprehensive education to CKD patients about the benefits of LDKT as early as possible can increase the chances that they will learn about it, seek living donors, and ultimately receive an LDKT [3]. Many patients, particularly socioeconomically disadvantaged patients and patients of racial/ethnic minority groups [4], have not had sufficient DDKT and LDKT education before their kidneys failed. [5, 6] Generally, patients not considering LDKT lack knowledge about the benefits of living donation over remaining on dialysis [7], have concerns about involving and risking a living donor's health [7], fear asking others to donate a kidney [8], or fear their own surgical pain and the possibility of the transplanted kidney failing [9].

While Black and Hispanic patients experience higher rates of diabetes and hypertension [5] and increased chances of developing ESRD versus their White or non-Hispanic counterparts [10], they are less likely to complete transplant evaluation $[6,11,12]$, experience longer wait-list times $[13,14]$, and are less likely to receive a DDKT or LDKT $[6,10,15,16]$. While $11.4 \%$ of White patients had received an LDKT after two years of being wait-listed, only 2.9 and $5.9 \%$ of Black and Hispanic patients, respectively, had received an LDKT $[11,17]$. The disparity in transplant knowledge between White patients and patients of racial or ethnic minorities has been well documented by our research team $[7,8,18,19]$.

Without a nationally coordinated healthcare system in the United States, it is difficult to ensure that patients moving from CKD Stages 3 and 4 to ESRD are consistently making informed transplant decisions. Limited research is available about how transplant education for patients in CKD Stage 3 and 4 is delivered. One study in community nephrologist's offices, the Talking About Live Kidney Donation (TALK) program, compared the efficacy of an LDKT print and video program, with or without in-person social worker discussions, on CKD patients' steps toward beginning transplant evaluation [20]. Though the TALK trial found that the discussion-oriented, social worker intervention had a higher predicted probability of taking additional steps in comparison to the education-only group, it also found that significantly higher proportions of patients in the education-only group took key steps towards transplant, like completing transplant evaluation.

Effective strategies for transplant education within dialysis centers are important to understand since $70 \%$ of ESRD patients are on dialysis [21], some of whom may never present to a transplant center for evaluation. Our team surveyed dialysis educators at 170 dialysis centers and found that $81 \%$ of educators were educating patients by recommending that they learn more about transplant by going to a transplant center or learning about transplant themselves [22]. In another study, only $24 \%$ (297 of 1223) of "informed" patients (based on patient-reported data submitted to the Centers for Medicare and Medicaid Services on Form-2728) reported that educators had detailed discussions about the risks and benefits of DDKT and LDKT with them [22]. Other similar studies have found that less than half of dialysis patients receive comprehensive discussions or counselling about the risks and benefits of transplant [22]. Our previous research has also shown that while kidney patients spend over $500 \mathrm{~h}$ in the dialysis center annually, they only spend a median of one hour reading brochures about transplant and 30 min talking about transplant with medical staff [4]. Only a minority of dialysis centers have formal education programs or provide transplant education to share with potential living donors [22-24]. For these and many other reasons, evidence suggests that not 
all dialysis patients receive appropriate information about transplant $[5,6,22,23,25]$.

Previous research has shown that two of the strongest predictors of successful completion of transplant evaluation are having access to more transplant education resources and having greater transplant knowledge at the onset of transplant evaluation, the latter also being the only significant predictor for ultimately receiving an LDKT [26]. With providers outside of transplant centers reporting constraints to educating patients about transplant [23], it is possible that providing supplementary education within a large health care system, with its fully integrated care management program, may be an opportune way to efficiently educate more CKD 3-5 patients about the opportunities for and risks and benefits of DDKT and LDKT. Further, the use of technology, such as text messaging, videos, and digital applications may reduce the provider burden barrier to delivering transplant education.

There is previous evidence that delivering more comprehensive education over time may be helpful to patients making informed decisions outside of transplant centers. The RaDIANT program in Georgia which targeted dialysis providers and patients with a multi-component educational intervention including patient educational toolkits, videos, and a transplant mentoring program over one-year found a $75 \%$ increased adjusted odds of referral for kidney transplant evaluation and that the intervention activities were more effective for Black versus White patients [27]. Through a group randomized controlled trial, a version of Explore Transplant (ET) delivered face-to-face over four meetings with patients while they were undergoing dialysis, was shown to increase patients' knowledge and informed decision-making significantly more than standard transplant education offered in dialysis centers [28]. Finally, Explore Transplant@Home (ET@Home) delivered by mail and supported through bimonthly postcards and texting over an eight-month period also found significant increases in transplant knowledge and informed decision-making among ESRD patients who received ET in this format, compared with standard transplant education in dialysis centers [29].

To date, ET and ET@Home have never been studied with patients who are in CKD Stages 3 \& 4 or with Asian or Spanish-speaking patients. This study is a partnership between UCLA and Kaiser Permanente Southern California (KPSC). KPSC's fully integrated care management program, diverse membership, and ability to track a large patient population offers a rare opportunity to study how knowledgeable CKD Stage 3-5 patients are about LDKT, assess disparities in knowledge across many races, ethnicities, and languages, and conduct a large-scale trial of the effectiveness of ET@Home. This manuscript will describe the protocol for a new randomized control trial (RCT) conducted in collaboration with KPSC to assess the effectiveness of ET@Home within a large healthcare system with a diverse group of CKD 3-5 patients.

\section{Study design}

The purpose of this study is to assess whether the ET@Home program can be integrated successfully across a large racially, ethnically, and geographically diverse patient catchment area and to assess its effectiveness to increase knowledge about the risks and benefits of LDKT and increase informed decision-making. The effectiveness of the ET@Home program will be evaluated in a randomized controlled trial (RCT) of 1200 Black, Hispanic, Asian, and White patients with stage 3, 4, or 5 CKD. Patients will be randomized to receive: (1) no additional education other than what is provided within KPSC (standard-of-care); or (2) a video-guided, four-part ET@Home program delivered by mail and supported through bimonthly postcards and texting over six months. The study has two aims.

\section{Aim 1}

Before intervention, to assess differences in knowledge and decision-making about the opportunities for and risks and benefits of living kidney donation for 1200 CKD Stage $3-5$ patients by race/ethnicity and primary language spoken.

\section{Hypothesis}

Patients earlier in the CKD continuum, patients who speak Spanish, and non-White patients will have less knowledge about the opportunities for and risks and benefits of living kidney donation and will be making less informed LDKT decisions.

\section{Aim 2}

To conduct a randomized controlled trial in English and Spanish of ET@Home for CKD Stage 3-5 patients to assess its effectiveness to increase LDKT knowledge and decision-making by race/ethnicity and primary language spoken as compared to the standard KPSC education.

\section{Hypothesis 1}

At the conclusion of the trial, CKD patients who receive ET@Home will have greater transplant knowledge and be more likely to make an informed treatment decision than patients receiving KPSC education alone.

\section{Hypothesis 2}

ET@Home will be more or equally effective for patients earlier in the CKD continuum, patients who speak Spanish, and non-White patients than patients in these subgroups receiving KPSC education alone. 


\section{Methods}

\section{Theoretical Foundation of ET@home}

The Explore Transplant (ET) education program was created based on Prochaska's Transtheoretical Model of Behavioral Change (TTM) and research with over 1000 patients with CKD, to address gaps in their transplant knowledge [30]. ET allows transplant-eligible patients to explore the option of remaining on dialysis or pursuing DDKT or LDKT and make an informed choice after knowing their benefits and risks. The TTM holds that patients vary widely in their levels of readiness to make important decisions about their health [31], like whether or not to get an LDKT [32]. This study will employ educational resources from the ET@Home program that are based on the TTM and that are designed to help CKD patients at all stages of readiness and decision making around DDKT and LDKT learn more about their treatment options and to ultimately choose the option best for them. The ET@Home educational resources never pressure patients to pursue transplant of any kind; rather, they provide accurate information about the complex set of potential risks and benefits that need to be considered when deciding whether to get a DDKT or LDKT, including potential increases in length and quality of life, resuming life activities, implications of taking transplant medications, the chances of injury to a living donor, and many more factors.

\section{Study participants: Eligibility, recruitment, and randomization}

KPSC provides coverage and care to nearly 65,000 patients with CKD Stages 3-5 throughout Los Angeles, San Diego, Kern, San Bernardino, Riverside, and Ventura Counties. About 24\% of these patients are Hispanic, 52\% White, 15\% Black, and 9\% Asian, similar to the broader CKD population in California. As of December 2015, more than 1500 KPSC CKD patients were listed on the United Network for Organ Sharing (UNOS) waiting list awaiting a DDKT, and approximately 700 additional KPSC patients were undergoing transplant evaluation. However, since 2014, only 350 KPSC patients have received a kidney transplant, with fewer than 100 patients receiving a LDKT.

Inclusion criteria for the study include adult KPSC patients between 18 and 70 years holding continuous membership with KPSC, currently being treated for CKD 3, 4, or 5/ESRD, having had at least one visit to a KPSC nephrologist in the last 18 months and who speak English or Spanish. To target enrollment of the CKD 3 patients at highest risk of worsening CKD, investigators will calculate a risk score for CKD 3 patients using the model described in Tangri et al. [33]

Exclusion criteria include having known medical or other permanent contraindications to transplant including cancer, heart failure, chronic obstructive pulmonary disease, hepatitis, cirrhosis, or dementia, or previous delisting or rejection for renal transplant by a transplant center. Other exclusion criteria include not being able to speak and read English or Spanish or having previously received a transplant. Those who have already refused to be evaluated for a transplant will also be excluded from the study. Patients' transplant eligibility will be confirmed by reviewing relevant information (e.g., absence of serious heart disease or cancer) from their Kaiser electronic medical record.

Prospective participants who meet eligibility criteria will be sent an email inviting them to enroll in the study and asked to complete the initial transplant decision-making survey online or on the phone. Study staff will invite patients to participate in the RCT, beginning at the top of the risk-score ranked list, moving down the list until stratified cell targets are met. Recruitment will be stratified by race/ethnicity, language spoken, and CKD stage, oversampling smaller subgroups within the KPSC patient population. Expecting a response rate of 20\%, an initial sample of 6000 patients will be invited to participate, resulting in a final sample of 1200 patients (Fig. 1). Clicking the survey link or contacting the study team by phone to begin the survey indicates consent to participate in the study. The email includes an opt out link if they do not wish to participate in the study. Invited prospective participants will receive follow-up invitations by secure email and phone to complete the initial transplant decision-making survey.

After completing the initial survey, enrolled participants will be randomized in a 1:1 ratio, stratified by race/ethnicity, language spoken, and CKD stage, to either the ET@Home intervention condition or standard KPSC education. The randomization sequence is produced electronically within the data capture system. Once an enrolled participant is assigned to an intervention arm, the study team will send the participant a notification of their assignment. Since this is an educational intervention, neither the study participants nor the study team are blinded to treatment assignment.

\section{Settings}

Since the CKD patients served by KPSC receive care in 13 KPSC centers spanning over 2000 mile $^{2}$ ranging across Los Angeles and San Diego metropolitan areas, an intervention strategy and setting that can reach all of these patients needs to be employed. Also, the intervention needs to reach racial and ethnic minority patients, who often have fewer transportation resources available to them and may be less able to travel even locally to take advantage of CKD education programs. Finally, the DDKT and LDKT education program needs to be flexible and capable of integration into the ongoing 


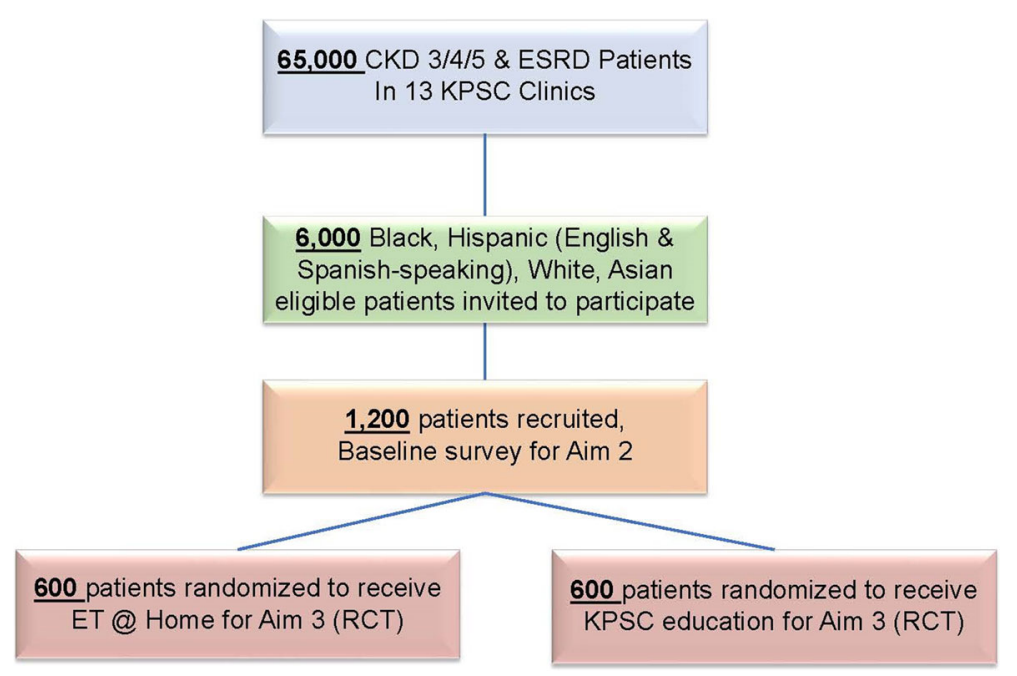

Fig. 1 Patient Recruitment Flowchart

transplant education and care management programs within KPSC so it does not create additional burden on busy transplant coordinators and patient educators. Thus, the setting for this Explore Transplant@Home trial will be guided learning within the patient's homes, for patients and any interested individuals in their social support networks, through use of video, print, and text education.

\section{Intervention (RCT educational components)}

\section{Description of standard KPSC transplant education (control)}

All KPSC nephrology departments currently offer a 1-2 $h$ course in transplant education to all CKD patients interested in pursuing transplant, whether from a living or deceased donor. The KPSC course addresses topics including how kidney transplantation works, determining whether kidney transplantation is the right choice, the steps involved in pre-transplant evaluation and referral to transplant centers of excellence. Because time is limited, the bulk of the content covered is oriented toward developing an understanding of the evaluation and wait-listing process, rather than on the risks and benefits of pursuing LDKT. Patients are encouraged to bring any prospective donors to the class with them, and course attendees learn about the basics of donor testing, some simple information regarding matching and donor exchanges, and reminders that LDKT can result in a much shorter wait for a donor organ. Written materials and presentations from the educators do recommend that patients seek out living donors but do not provide details on how to approach prospective donors. The materials address only the risks and benefits to the recipient not to the donors. Compared to DDKT, much less detailed information is provided regarding LDKT, unless prompted by interested patients. Practical information about waiting for a transplant, including local wait-list times and allocation criteria, the transplant procedure, and steps for post-graft follow-up are covered in detail. KPSC educators include nephrology nurses and social workers. The course is presented monthly in English with available real-time language interpreters at each of 13 local medical centers, in Spanish at 1-2 centers, and is supplemented by a written pamphlet in English and Spanish.

While patients at all phases of CKD and their family members and support persons are welcome to attend the transplant education course, it is primarily focused on individuals who have reached an advanced stage of CKD and/or ESRD and are contemplating renal replacement options, or who are new to dialysis and considering transplant as a renal replacement approach. Patients who wish to be evaluated for transplant eligibility and move forward with the process of pre-transplant assessment and listing are required to complete this course before beginning pre-transplant work-up. For this trial, routine transplant education practices will be continued without any content changes. Patients who are randomized to the control condition and who are or become potentially eligible for transplant will not receive any additional interventions within KPSC during the study period.

\section{Description of patient-guided Explore Transplant@Home program}

Patients in the ET@Home study condition will receive four modules of video and print transplant education over a 6-month period (Table 1). The modules sequentially help patients think through what is important to them, learn what it might be like if they became a kidney recipient or involved a living donor, the risks and 

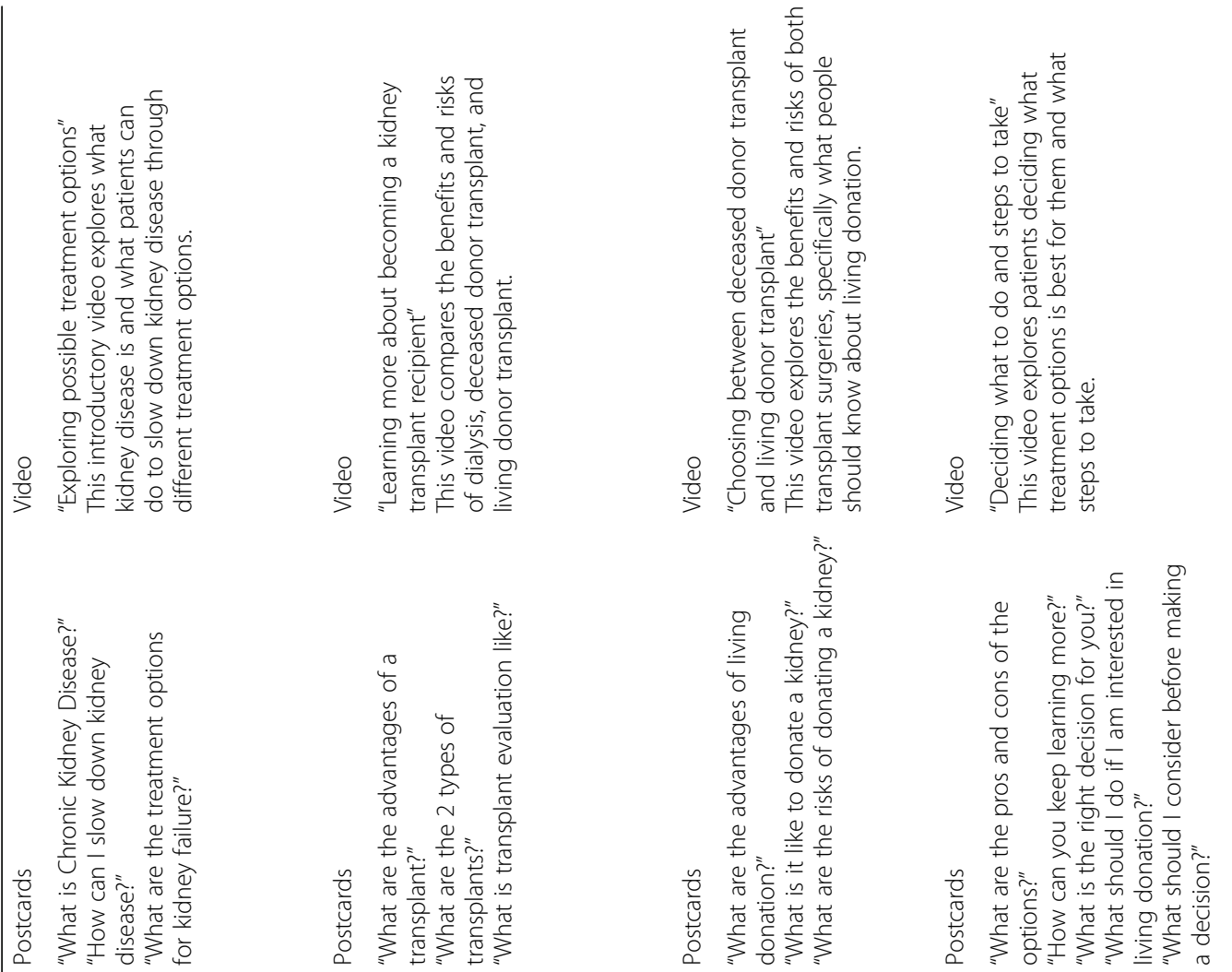

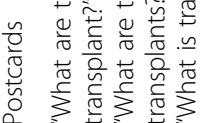
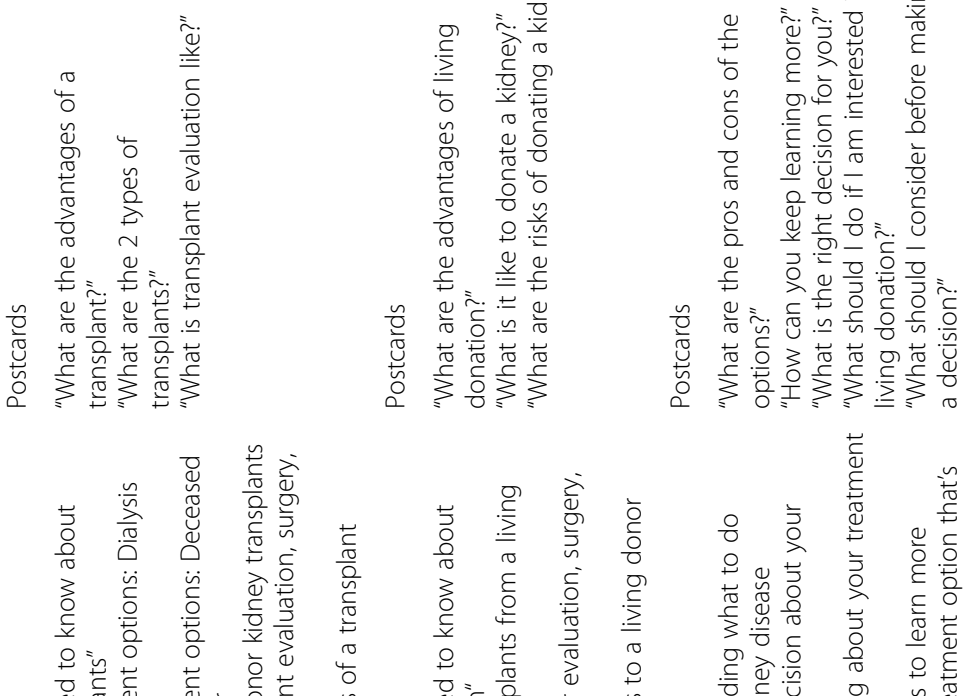

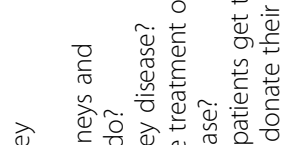

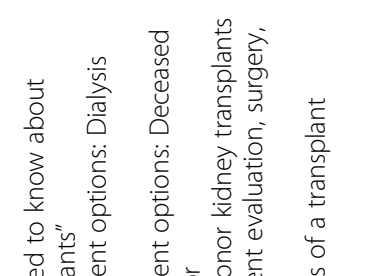

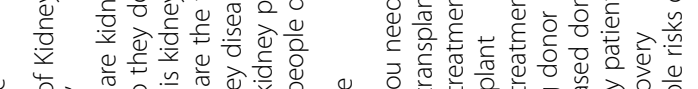

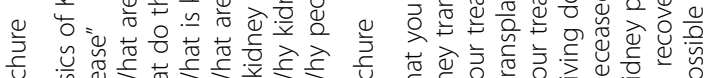

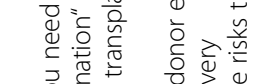

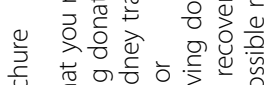

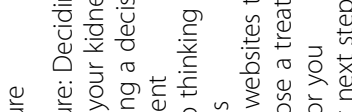

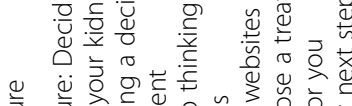

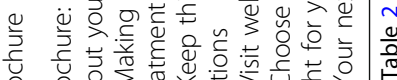

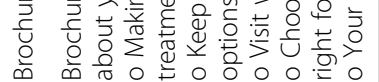
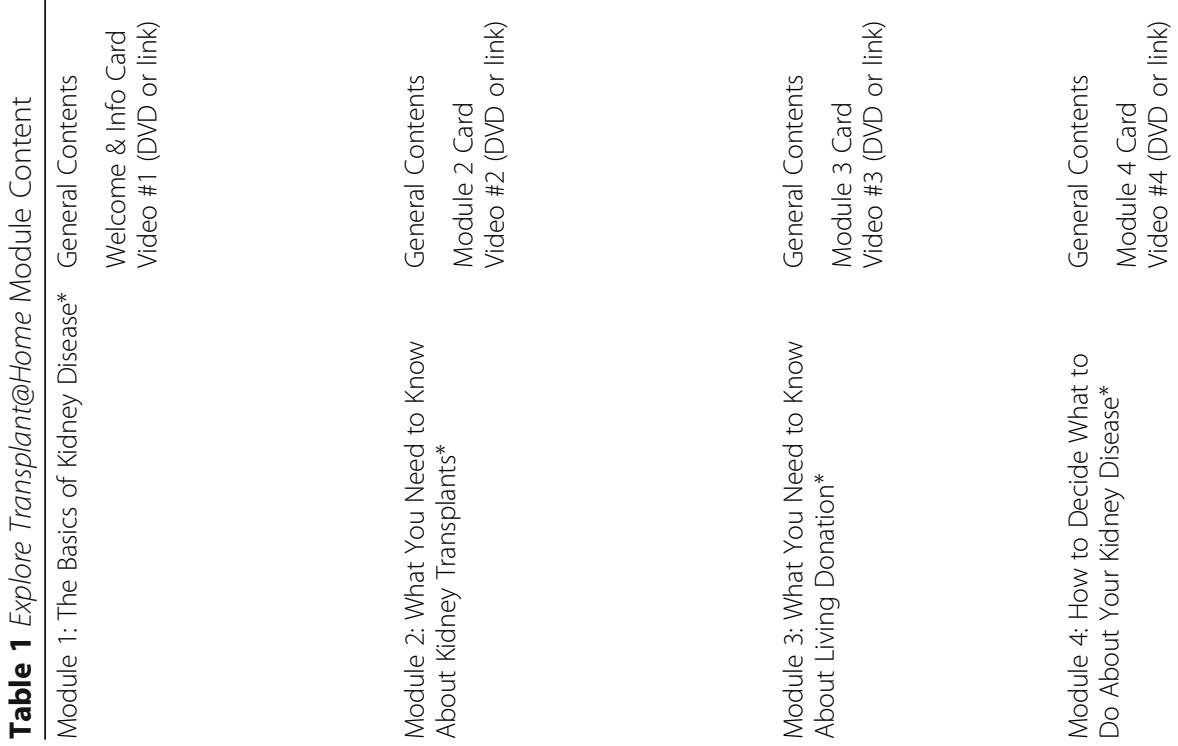

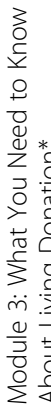

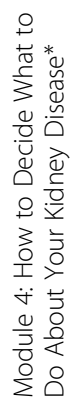


benefits of each, and, at the program's conclusion, make a decision about the best treatment option for them. The four videos and their corresponding print brochures "Exploring Transplant", "Kidney Recipients' Transplant Experiences," "Living Donors' Donation Experiences," and "Making an Informed Choice" - discuss in detail the medical, psychological, and financial risks of being a deceased or living donor. These modules cover ways to slow down kidney disease and include real life quotes and stories from people who have stayed on dialysis, received DDKT and LDKT, or been a living donor. The "Making an Informed Choice" brochure is a decision aid that guides patients in choosing a path that is right for them and provides helpful websites that patients can use as resources to learn more. At the conclusion of the program, patients individually choose which renal replacement treatments may be right for them and plan a set of small steps they want to take next.

To expand this program for patients in CKD 3 and 4, we conducted a combination of 32 additional focus groups and individual interviews to learn what additional content should be added. Based on recommendations, we added more content about the purpose and function of kidneys, explaining in detail what kidney disease is and its various stages, how to slow down the kidney failure process, and providing an overview of treatment options once the patient enters kidney failure. Experts from the nonprofit organization, Health Literacy Media, and the study investigators reviewed and revised all print content using the most current, evidence-based health literacy principles [34]. The review and edits included considerations around audience appropriateness, readability, behavioral orientation, interactivity, information architecture, plain language, and clear design.

Each Explore Transplant@Home video begins and ends with a transplant educator and kidney professional or patient introducing the video and then making specific recommendations afterwards about what to think about next. The healthcare professionals on the video are diverse in their gender and ethnicity. On the final video of the series, a patient and his wife models meeting with the educator to work through the decision-aid and make a decision that is right for him or her. This modeling occurs on the video to support the patient watching and doing the same at home with his family. The Spanish language version of the video (voiced in Spanish, not subtitled) has been awarded a certificate of translation.

During the baseline survey, patients will be asked about the availability of Internet and DVD resources in their home. If the patient does not have a DVD player, a DVD player will be provided as a service of the grant. Should they prefer to watch this program via Internet streaming, an individualized login code will be provided to them. Individual login data for patients utilizing the Internet will be tracked to determine fidelity.

After each module of print and video education is mailed, postcards will be mailed weekly, recapping important content covered within the videos and brochures. Patients will have the opportunity to participate in a texting component of ET@Home that also sends educational content through learning reminders and questions by SMS text message each week (Table 2). Study participants will receive text messages daily that include study participation reminders, motivations to continue in the study, CKD facts, recommendations for learning more, and quiz questions to test knowledge. Participation in the text messaging portion of the trial is voluntary, and the option to opt out is available at all times.

If patients have questions for their kidney and transplant providers, they are directed to contact the ET@Home Help line, which can connect them with their KPSC providers for more information.

\section{Involving social support network}

Our general recommendation through the program will be for patients to involve their family and friends in learning and decision-making about transplant. The brochures also recommend that the patient watch the video with other people in their lives who help them make important health decisions, particularly the stories of living donors, and complete the decision aid with them.

\section{Variables}

Before the intervention, a baseline patient survey will measure the following independent variables: participants' demographic characteristics, socioeconomic derailers to

Table 2 Examples of ET@Home Text Messages

\begin{tabular}{ll}
\hline REMINDER & Your first packet "Basics of Kidney Disease" is headed your way! Keep an eye out for the postcards for this packet as well. \\
MOTIVATION & You can slow down how fast your kidneys fail. Learn more about all of your options! \\
FACT & Did you know that the kidneys help keep bones healthy? \\
RECOMMENDATION & $\begin{array}{l}\text { Chronic kidney disease (CKD) can present itself through a variety of symptoms. It's important that you familiarize yourself with } \\
\text { these symptoms so you can track changes and talk to your doctor. }\end{array}$ \\
QUIZ QUESTION & $\begin{array}{l}\text { Can you slow down how fast your kidneys fail? Reply with YES or NO. } \\
\text { ANSWER FOR YES }\end{array}$ \\
\hline
\end{tabular}


pursuit of transplant, medical mistrust, health-related quality of life (HRQOL), health literacy, and the amount of previous transplant education they have received. It will also assess the following dependent variables: level of current transplant knowledge, current ability to make an informed decision about transplant, self-efficacy to pursue DDKT and LDKT, and steps taken toward transplant. After the intervention, a post-survey will measure key study dependent variables to assess change, including the level of current transplant knowledge, current ability to make an informed decision about transplant, self-efficacy to pursue DDKT and LDKT, and steps taken toward transplant. For patients randomized to receive the ET@Home education, it will also assess their experiences with the educational materials through a program evaluation assessment.

Outcome measures and research methodology In order to verify and demonstrate impact on improving knowledge of the process, risks, advantages, and disadvantages of deceased and living donation among dialysis patients, an RCT will be conducted. In this RCT, 1200 CKD patients in the KPSC system will be randomized to one of two education conditions (patient-guided ET@Home vs. standard KPSC transplant education, 600 patients per condition). Changes in key outcomes will be assessed comparing patient data collected at baseline and six months post-baseline. The primary outcome measures include patients' knowledge of CKD and the risks and benefits of transplant and the capacity to make an informed decision about transplant. Additionally, key secondary measures will be examined, including self-efficacy to pursue DDKT and LDKT and steps taken towards transplant.

Comparisons in the two educational conditions' effectiveness will also occur for patients earlier in the CKD continuum, patients who speak Spanish, and non-White patients.

Methodology Patients will be remunerated $\$ 20$ with a gift card after completing a baseline survey. Most surveys will be completed online by the study participants directly or over the phone with a trained interviewer. Any mailed surveys will be returned to KPSC and entered into the study's assessment database. A double-data entry method will be used to ensure accurate data entry. Separate databases will be created for each patient's registration/contact information and assessment data. Each patient will be given a unique, arbitrary study identification number that can be used to associate their contact and registration information and assessment data. Once the 1200 surveys have been collected and entered, this data will be analyzed to determine baseline differences in transplant knowledge, informed decision-making, self-efficacy, and steps toward transplant between patients varying in race/ethnicity, primary language, and CKD stage before patients are given an intervention.

\section{Evaluation and statistical analysis plan Power analysis}

Based on the following assumptions, we estimated the sample size for the RCT. Using data from previous RCTs $[4,35]$, a mean knowledge change score of 1.0 points is estimated necessary to be detected among patients receiving ET@Home and standard KPSC education. Patients' baseline transplant knowledge scores will be correlated with those of other patients from the same clinics [intraclass correlation coefficient (ICC) of 0.31]. The variance in knowledge change has both betweenand within-cluster components, yielding a pooled standard deviation estimate of 4.0. Participant retention will be $50 \%$ at the 6 -month assessment. Outcomes will be assessed using two-tailed statistical tests. Type 1 error rate (alpha level) will be set at 0.05 . This study design will ensure power of 0.90 . Power calculations for this trial are based on all of these factors, which yields a standardized effect size, accounting for clustering of approximately 0.257 .

Power analyses based on changes in transplant knowledge, our primary study outcome, are presented. The study design and analyses were treated as a test of the difference between the mean knowledge score change of CKD patients in the ET@Home and standard KPSC education (control) conditions six months post-baseline. It is estimated that with 600 completed surveys (50\% attrition), there will be a $90 \%$ power to detect a 0.25 effect size between two equal-sized groups (control and treatment groups). By stratifying recruitment and randomization by race/ethnicity (Asian, Black, Hispanic, White), primary language spoken (English vs. Spanish), and stage of CKD, according to the table below, this sample size will also have $90 \%$ power to detect 1 point differences in change on the transplant knowledge scale between the ET@Home and standard KPSC education conditions for these critical subgroups using interaction terms (e.g., treatment by race interaction, language by race interaction). Patients enrolled and randomized are shown in Table 3.

\section{Measures}

Demographic and clinical characteristics

Patient demographics, including patients' gender, age, race/ethnicity, preferred language, and CKD stage will be obtained from the KPSC electronic medical record. 
Table 3 Patients enrolled and randomized

\begin{tabular}{lllll}
\hline Race/Ethnicity/Language & \multicolumn{5}{l}{ Stage of CKD } \\
\cline { 2 - 5 } & $3^{\text {a }}$ & 4 & $5 /$ ESRD & Total \\
\hline English-speaking Asian & 67 & 67 & 66 & 200 \\
English-speaking Black & 67 & 66 & 67 & 200 \\
English-speaking White & 67 & 67 & 67 & 200 \\
English-speaking Hispanic & 67 & 67 & 66 & 200 \\
Spanish-speaking Hispanic & 133 & 133 & 134 & 400 \\
Total & 400 & 400 & 400 & 1200 \\
\hline
\end{tabular}

${ }^{a} C K D$ patient recruitment prioritized based on likelihood to progress

\section{Kidney transplant Derailers index}

Measures common transplant derailers such as level of education, health literacy, financial stability, neighborhood/environmental safety, access to transportation, and social support. Health literacy will be assessed with two items measuring subjective health literacy that were shown to detect health literacy as well as gold standard measures such as the S-TOHFLA and the REALM [36].

\section{Global Health-related quality of life}

Global HRQOL will be measured with the PROMIS 10-item global health scale [37], which produces summary physical and mental scores, each of which has 4 items supported by factor analyses and IRT analyses, with good reliabilities found: physical $=0.81$, mental $=$ 0.86 .

\section{Previous transplant education}

The measure of previous transplant education captures the quantity and quality of transplant education, and it has been correlated with taking action toward pursuing transplant in previous studies [26].

\section{Small steps}

A measure of whether patients took any of 25 steps toward transplant (e.g., "Do you plan to talk through your treatment options with people you trust?" or "Do you plan to call the transplant center to begin evaluation?"). At each survey, patients will be asked whether they had "Already done," "Plan to do," or "Don't plan to do" each step. These questions will be completed at baseline and six months.

\section{Level of medical mistrust}

Medical Mistrust will be measured with the Medical Mistrust Index (MMI) [38]. This scale was supported by factor analysis and exhibits moderately strong test-retest reliability, with correlation coefficients ranging from $0.3-0.7$.

\section{LDKT and DDKT self-efficacy}

The factor structure for the LDKT scale was verified with exploratory and confirmatory factor analyses, demonstrating a good fit for a one-factor solution (loadings ranging between 0.61-0.91) [32]. The Cronbach's alpha was 0.88 , indicating a high level of internal reliability. The factor structure of the DDKT scale was also verified with exploratory and confirmatory factor analyses, demonstrating a good fit for a one-factor solution (loadings ranging between $0.50-0.73$ ) [39]. The alpha was 0.85 , indicating good internal consistency reliability. Self-efficacy will be measured at baseline and six months.

\section{CKD, LDKT and DDKT knowledge}

A knowledge scale was created using items our team has employed in several previous studies and legacy measures $[6,35,40]$ and new questions in response to issues raised by patients and clinicians. A scale created from the extant items has been evidenced with factor analyses [41]. Knowledge will be measured at baseline and six months.

\section{Informed decision-making}

An informed decision-making measure was created from legacy measures and items generated from our formative work. Items for this scale were selected from legacy scales with good psychometric properties, like the Decisional Conflict Scale, whose total scale and subscales evidence high internal consistency, with Cronbach's alphas ranging between 0.6-0.9. There was not a statistically significant difference between test-retest scores for this scale, and there was a high correlation $(r=0.80)$ between them [42]. Informed decision-making will be measured at baseline and six months.

\section{Process evaluation}

At the six-month follow-up assessment, all intervention participants will complete an evaluation of the helpfulness of and their satisfaction with the print and video materials, text messages, and the ET@Home program as a whole.

\section{Statistical analysis}

The available demographic and clinical characteristics of patients who refuse to join the study will be compared to those who enroll to determine if the patient selection procedure has resulted in a biased sample. A similar analysis will be conducted to compare the patients who fail to complete the six-month assessment. Multiple imputation by chained equations (MICE) will be used to multiply impute missing data. Imputation models will include all analysis and stratification variables. The intent-to-treat (ITT) approach will be applied in that all 
study participants will be analyzed according to the group that they were randomized to, regardless of whether they actually received their assigned intervention or not. Responses to the six-month intervention evaluation will be summarized for the sample overall and by levels of the stratification factors.

It is hypothesized that patients earlier in the CKD continuum, patients who speak Spanish, and non-White patients will have less knowledge about the opportunities for and risks and benefits of living kidney donation and will be making less-informed LDKT decisions. Investigators will evaluate these hypotheses using data from the pre-survey (baseline) by fitting multilevel random effects models with a random intercept to account for clustering at the CKD clinic level to compare differences in knowledge, informed decision-making, and Self-Efficacy. First, unadjusted models will be examined with the race/ethnicity, primary language spoken, and stage of CKD as independent variables for each test (separate models for each). Then, each of these models will be adjusted for other factors hypothesized to impact each outcome, including level of socioeconomic vulnerability, the level of previous transplant education received, the level of medical mistrust, and the level of health literacy. The addition of these variables will help test and control for confounding.

Next, the effectiveness of ET@Home for CKD Stage 35 patients to increase LDKT knowledge and decisionmaking compared to KPSC education, by race/ethnicity and primary language spoken, will be evaluated. First, it will be determined if baseline differences in patient- and clinic-level variables are present using $\chi^{2}$ and Wilcoxon rank-sum tests, and subsequently adjustments for any out-of-balance characteristics in outcome analyses will be made. Differences in changes in transplant knowledge and Self-Efficacy using change scores, defined as the difference between the baseline and post-survey assessments, will be examined. Differences in these outcomes between the educational conditions will be tested using multilevel random effects models with normal outcome distributions, accounting for clustering with a random intercept for CKD clinic. Differences in informed decision-making will be examined using multilevel random effects logistic models. Finally, for the number of new steps taken, differences between the educational conditions in the count of new steps will be analyzed with a multilevel random effects Poisson model.

After the primary analyses are completed, multilevel random effects models will be constructed to examine the heterogeneity of treatment effect (HTE) using interaction terms for the ET@Home educational condition by the key patient subgroups by which randomization was stratified, including race, primary language spoken, and stage of CKD. For example, the ET@Home educational condition (ET@Home vs. SOC) x primary language (English vs. Spanish) interaction term will be tested, enabling us to determine of the ET@Home intervention was more or less effective for primarily Spanish-speaking patients than English-speaking patients.

\section{Discussion}

Previous research has shown that improved transplant education, both inside and outside of transplant centers, can increase patients' knowledge of DDKT and LDKT, pro-transplant attitudes, referral for transplant, and LKDT rates [20, 27-29]. However, this RCT will allow us the opportunity and ability to partner with KPSC, a large healthcare system with a diverse patient base, to compare ET@Home with their current educational standard-of-care. We also will be able to assess a significant amount of data from a fully integrated care management program, diverse membership, and ability to track a large patient population as they make treatment decisions along the most coordinated care system in the country.

The four-part modular ET@Home program, delivered to CKD 3-5 patients through video, print, and texting systems over six months, is one of the first examinations of supplementary education that does not involve healthcare providers. This intervention also applies health literacy and transplant education best practices recommended for programs outside of transplant centers to deliver education directly to patients [34, 43, 44].

In addition, this RCT will examine the effectiveness of this program on patients of various races, stages of CKD, and primary languages spoken. The findings of this trial will foster greater understanding of how ExploreTransplant@Home specifically affects White, Black and Hispanic patients' LDKT decision-making, knowledge, and behavior. Additionally, as it will serve patients whose primary language is English and Spanish, it will allow us to learn more about their interest in preemptive transplant, DDKT, and LDKT. We hope this program will reduce disparities in access to transplant for patients who speak Spanish and for Black, Hispanic, and Asian patients served by KPSC.

Previous studies have identified low SES as a barrier to transplant, with patients of low SES being less likely to be wait-listed [45, 46]. Explore Transplant@Home is unique in its ability to study the impact of SES on patients' pursuit of LDKT. While previous studies have focused on SES factors that are difficult to modify $[13,47]$ or on the neighborhood or census block level [16, 47, 48],ET@Home will assess key, specific SES barriers and provide support services to address these barriers. This study will allow us to 
identify the most common SES-related influences challenging completion of transplant evaluation and getting an LDKT.

In conclusion, through this trial, investigators will gain an understanding of key knowledge gaps for patients along the CKD continuum and between patients who speak different languages. An RCT examining the effectiveness of both English- and Spanish-language ET@Home programs in increasing KPSC patients' knowledge about the opportunities for, and risks and benefits of, LDKT and making informed transplant decisions will be completed. Investigators will have a clear understanding of the facilitators and barriers occurring within the KPSC system to the delivery of transplant and LDKT education for patients of varying racial and socioeconomic groups. If successful, the dissemination approach of ET@Home could easily and immediately be expanded to other CKD patients within KPSC and Kaiser Permanente Northern California. The lessons learned and approach to disseminating this low-costET@Home educational approach could be utilized within other healthcare and insurance companies, serving hundreds of thousands of diverse patients to assist in their informed decision-making for DDKT and LDKT before and after their kidneys fail. We hope this ET@Home program will help to reduce disparities in access to transplant and creates a model to be replicated for other healthcare systems.

\footnotetext{
Abbreviations

AST: American Society of Transplantation; CKD: Chronic kidney disease; DDKT: Deceased donor kidney transplant; ESRD: End-stage renal disease; ET: Explore Transplant; ET@Home: Explore Transplant@Home; HRQOL: Healthrelated quality of life; HRSA: Health Resources and Services; ICC: Intraclass correlation coefficient; KPSC: Kaiser Permanente Southern California; LDKT: Living donor kidney transplant; MMI: Medical Mistrust Index; RCT: Randomized control trial; RRT: Renal replacement therapy; TTM: Transtheoretical Model of Behavioral Change; UNOS: United Network for Organ Sharing
}

\section{Acknowledgements}

Thank you to Jenny Burman for her assistance in preparing and editing this manuscript, as funded by the Terasaki Research Institute.

\section{Funding}

This project received support from the NIH NCATS UCLA CTSI, Grant Number UL1TR001881, and from the Health Resources and Services Administration, Grant Number R390T29879.

The funders had no role in study design, data collection and analysis, decision to publish, or preparation of the manuscript.

\section{Availability of data and materials}

The datasets produced after the completion of this trial may be requested from the principal investigator (ADW).

\section{Authors' contributions}

All authors read and approved the final manuscript, and all authors made intellectual contributions to the design of the trial. ADW, AFL, CSA, and JLB prepared the manuscript. $\mathrm{AA}, \mathrm{BD}, \mathrm{SH}$, and $\mathrm{BM}$ revised the manuscript.

\section{Ethics approval and consent to participate}

This protocol was approved by the IRB committee of the University of California, Los Angeles and Kaiser Permanente Southern California. The IRB protocol numbers are 11469 (Kaiser Permanente) and 18-001099 (University of California, Los Angles). Consent to participate is provided verbally, as approved by the IRBs of both institutions.

\section{Consent for publication}

This article does not contain any personally identifiable information from any participants.

\section{Competing interests}

Dr. Amy D. Waterman, PhD owns the intellectual property to the transplant education product Explore Transplant and has licensed it at no-cost to a nonprofit, Health Literacy Media (HLM), who retains all revenue as to their sales. She serves as an unpaid consultant to HLM to ensure the accuracy of educational content. All other authors declare that they have no competing interests.

\section{Publisher's Note}

Springer Nature remains neutral with regard to jurisdictional claims in published maps and institutional affiliations.

\section{Author details}

${ }^{1}$ Division of Nephrology, David Geffen School of Medicine, University of California Los Angeles, Los Angeles, California, USA. ${ }^{2}$ Terasaki Research Institute, Terasaki Research Institute, 1018 Westwood Blvd, Los Angeles, CA 90024, USA. ${ }^{3}$ Kaiser Permanente Southern California, 100 S Los Robles Ave,

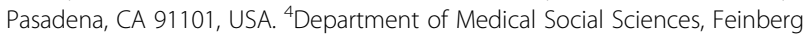
School of Medicine, Northwestern University, Chicago, USA.

Received: 9 November 2018 Accepted: 20 February 2019

Published online: 04 March 2019

\section{References}

1. United States Renal Data System. 2015 annual data report: An overview of the epidemiology of kidney disease in the United States. Bethesda: National Institute of Health, National Institute of Diabetes and Digestive Diseases; 2015

2. Neipp M, Karavul B, Jackobs S, Meyer ZU, Vilsendorf A, Richter N, Becker T, Schwarz A, Klempnauer J. quality of life in adult transplant recipients more than 15 years after kidney transplantation. Transplantation. 2006; 81(12):1640-4.

3. Patzer RE, Perryman JP, Pastan S, Amaral S, Gazmararian JA, Klein M, Kutner $\mathrm{N}$, McClellan WM. Impact of a patient education program on disparities in kidney transplant evaluation. Clin J Am Soc Nephrol. 2012;7(4):648-55.

4. Waterman AD, Hyland SS, Stanley SL, Barrett AC, Millinger R. Improving education increases dialysis patients' pursuit of transplant: Explore Transplant RCT findings. In: American Transplant Congress: May 30-June 3 2009. Boston: American Journal of Transplantation; 2009. p. 360

5. Kutner NG, Zhang R, Huang $Y$, Johansen $\mathrm{KL}$. Impact of race on Predialysis discussions and kidney transplant preemptive wait-listing. Am J Nephrol. 2012;35(4):305-11.

6. Waterman AD, Peipert JD, Hyland SS, McCabe MS, Schenk EA, Liu J. Modifiable patient characteristics and racial disparities in evaluation completion and living donor transplant. Clinical journal of the American Society of Nephrology : CJASN. 2013;8(6):995-1002.

7. Waterman AD, Barrett AC, Stanley SL, Waterman BM, Burroughs TE, Highstein G, Rodrigue JR, Hong BA, Brennan DC: Psychosocial and knowledge barriers preventing renal patients from pursuing living and deceased donor transplantation. In: World Transplant Congress: July 22 2006; Boston: American journal of transplantation; 2006.

8. Waterman AD, Barrett AC, Stanley SL. Optimal transplant education for recipients to increase pursuit of living donation. Prog Transplant. 2008; 18(1):55-62.

9. Waterman AD, Stanley SL, Covelli T, Hazel E, Hong BA, Brennan DC. Living donation decision making: recipients' concerns and educational needs. Prog Transplant. 2006;16(1):17-23.

10. USRDS 2013 Annual Report: Atlas of Chronic Kidney Disease and End-Stage Renal Disease in the United States. [http://www.usrds.org/].

11. Weng FL, Joffe MM, Feldman HI, Mange KC. Rates of completion of the medical evaluation for renal transplantation. Am J Kidney Dis. 2005; 46(4):734-45.

12. Lunsford SL, Simpson KS, Chavin KD, Menching KJ, Miles LG, Shilling LM, Smalls GR, Baliga PK. Racial disparities in living kidney donation: is there a 
lack of willing donors or an excess of medically unsuitable candidates? Transplantation. 2006;82(7):876-81.

13. Kasiske BL, London W, Ellison MD. Race and socioeconomic factors influencing early placement on the kidney transplant waiting list. J Am Soc Nephrol. 1998;9(11):2142-7.

14. Hall YN, Choi Al, Xu P, O'Hare AM, Chertow GM. Racial ethnic differences in rates and determinants of deceased donor kidney transplantation. J Am Soc Nephrol. 2011;22(4):743-51.

15. Kasiske BL, Snyder JJ, Matas AJ, Ellison MD, Gill JS, Kausz AT. Preemptive kidney transplantation: the advantage and the advantaged. J Am Soc Nephrol. 2002;13(5):1358-64.

16. Patzer RE, Perryman JP, Schrager JD, Pastan S, Amaral S, Gazmararian JA, Klein M, Kutner N, McClellan WM. The role of race and poverty on steps to kidney transplantation in the southeastern United States. Am J Transplant. 2012;12(2):358-68.

17. Purnell TS, Luo X, Cooper LA, Massie AB, Kucirka LM, Henderson ML, Gordon EJ, Crews DC, Boulware LE, Segev DL. Association of Race and Ethnicity with Live Donor Kidney Transplantation in the United States from 1995 to 2014 Jama. 2018;319(1):49-61.

18. Waterman A, Peipert J, Brann S, Kynard-Amerson C, Robbins M, Paiva A, Gaber A. Educating the less prepared: transplant knowledge and decisionmaking differences by race at onset of evaluation [abstract]. In: American Transplant Congress. Vol. 15. Philadelphia: American Journal of Transplantation; 2015.

19. Waterman A, Peipert J, Kynard-Amerson C, McSorley A-M, Paiva A, Robbins $M$, Peace L. Controlling for socioeconomic barriers reduces racial disparities in transplant pursuit. In: American Transplant Congress. Vol. 15. Philadelphia: American Journal of Transplantation; 2015.

20. Boulware LE, Hill-Briggs F, Kraus ES, Melancon JK, Falcone B, Ephraim PL, Jaar BG, Gimenez L, Choi M, Senga M, et al. Effectiveness of educational and social worker interventions to activate patients' discussion and pursuit of preemptive living donor kidney transplantation: a randomized controlled trial. Am J Kidney Dis. 2013;61(3):476-86

21. United States Renal Data System. 2016 USRDS annual data report: Epidemiology of kidney disease in the United States. Bethesda: National Institutes of Health, National Institute of Diabetes and Digestive and Kidney Diseases; 2016

22. Waterman AD, Peipert JD, Goalby CJ, Dinkel KM, Xiao H, Lentine KL. Assessing transplant education practices in Dialysis centers: comparing educator reported and Medicare data. Clin J Am Soc Nephrol. 2015;10(9):1617-25.

23. Balhara KS, Kucirka LM, Jaar BG, Segev DL. Disparities in provision of transplant education by profit status of the dialysis center. Am J Transplant. 2012;12(11):3104-10

24. Browne T, Patzer RE, Gander J, Amamoo MA, Krisher J, Sauls L, Pastan S. Kidney transplant referral practices in southeastern dialysis units. Clin Transpl. 2016;30(4):365-71.

25. Kucirka LM, Grams ME, Balhara KS, Jaar BG, Segev DL. Disparities in provision of transplant information affect access to kidney transplantation. Am J Transplant. 2012:12(2):351-7.

26. Waterman AD, Hyland SS, Liu J, Peipert J, Schenk EA, McCabe M. Understanding Racial Disparities and Effective Predictors of Successful Completion of Transplant Evaluation. In: American Transplant Congress. vol. 12. Boston: American Journal of Transplantation; 2012. p. 46.

27. Patzer RE, Paul S, Plantinga L, Gander J, Sauls L, Krisher J, Mulloy LL, Gibney EM, Browne T, Zayas CF et al: A Randomized Trial to Reduce Disparities in Referral for Transplant Evaluation. J Am Soc Nephrol 2017, 28(3):935-942.

28. Amy D, Waterman P, John D, Peipert P. An explore transplant group randomized controlled education trial to increase Dialysis patients' decisionmaking and pursuit of transplantation. Prog Transplant. 2018;28(2):10.

29. Waterman A, Peipert J, Xiao H, Goalby C, Lentine K: Educational Strategies for Increased Wait-listing Rates: Opportunities for Dialysis Center Intervention. In: American Transplant Congress. Chicago, IL; 2017.

30. Glanz K, Rimer BK, Viswanath K. Theory, Research, and Practice in Health Behavior and Health Education. In: Glanz K, Rimer BK, Viswanath K, editors. Health Behavior and Health Education. 4th ed. San Francisco: CA: JosseyBass; 2008. p. 23-38.

31. Prochaska JO, Norcross JC. Stages of change. Psychother Theory Res Pract Train. 2001;38(4):443-8.

32. Waterman AD, Robbins ML, Paiva AL, Peipert JD, Davis LA, Hyland SS, Schenk EA, Baldwin KA, Amoyal NR. Measuring kidney patients motivation to pursue living donor kidney transplant: development of stage of change, decisional balance and self-efficacy measures. J Health Psychol. 2015;20(2):210-21.

33. Tangri N, Inker LA, Hiebert B, Wong J, Naimark D, Kent D, Levey AS. A dynamic predictive model for progression of CKD. Am J Kidney Dis. 2017; 69(4):514-20.

34. Skelton SL, Waterman AD, Davis LA, Peipert JD, Fish AF. Applying best practices to designing patient education for patients with end-stage renal disease pursuing kidney transplant. Prog Transplant. 2015;25(1):77-84.

35. Waterman AD, McSorley AM, Peipert JD, Goalby CJ, Peace LJ, Lutz PA, Thein JL. Explore transplant at home: a randomized control trial of an educational intervention to increase transplant knowledge for black and white socioeconomically disadvantaged dialysis patients. BMC Nephrol. 2015;16:150.

36. Chew LD, Griffin JM, Partin MR, Noorbaloochi S, Grill JP, Snyder A, Bradley KA, Nugent SM, Baines AD, Vanryn M. Validation of screening questions for limited health literacy in a large VA outpatient population. J Gen Intern Med. 2008;23(5):561-6.

37. Hays RD, Bjorner JB, Revicki DA, Spritzer KL, Cella D. Development of physical and mental health summary scores from the patient-reported outcomes measurement information system (PROMIS) global items. Qual Life Res. 2009;18(7):873-80

38. LaVeist TA, Isaac LA, Williams KP. Mistrust of health care organizations is associated with underutilization of health services. Health Serv Res. 2009; 44(6):2093-105.

39. Waterman A, Robbins M, Paiva A, Hyland SS. Kidney patients' intention to receive a deceased donor transplant: development of stage of change, decisional balance, and self-efficacy measures. J Health Psychol. 2010;15(3):436-45.

40. Waterman $A D$, Robbins $M L$, Paiva AL, Peipert JD, Kynard-Amerson CS, Goalby C, Davis L, Thein J, Schenk E, Baldwin KA et al: Your path to transplant: a randomized controlled trial of a tailored computer education intervention to increase living donor kidney transplant. BMC Nephrol. 2014:15(166). https://doi.org/10.1186/1471-2369-15-166.

41. Peipert J, Weng F, Schenk E, Hyland S, Holland B, Brown DR, Waterman A. Development and validation of the "knowledge about renal transplantation" scale (KART). In: World transplant congress, vol. 14. San Francisco: American Journal of Transplantation; 2014. p. 196-08.

42. Wallston KA, Wallston BS, DeVellis R. Development of the multidimensional health locus of control (MHLC) scales. Health Educ Monogr. 1978;6(2):160-70.

43. LaPointe RD, Hays R, Baliga P, Cohen DJ, Cooper M, Danovitch GM, Dew MA, Gordon EJ, Mandelbrot DA, McGuire S, et al. Consensus conference on best practices in live kidney donation: recommendations to optimize education, access, and care. Am J Transplant. 2015;15(4):914-22.

44. Rodrigue $\mathrm{JR}$, LaPointe RD, Hays R. Living donor kidney transplantation: best practices in live kidney donation-recommendations from a consensus conference. Clin J Am Soc Nephrol. 2015.

45. Patzer RE, McClellan WM. Influence of race, ethnicity and socioeconomic status on kidney disease. Nat Rev Nephrol. 2012;8(9):533-41.

46. Schold JD, Gregg JA, Harman JS, Hall AG, Patton PR, Meier-Kriesche HU. Barriers to evaluation and wait listing for kidney transplantation. Clin J Am Soc Nephrol. 2011;6(7):1760-7.

47. Patzer RE, Amaral S, Klein M, Kutner N, Perryman JP, Gazmararian JA McClellan WM. Racial disparities in pediatric access to kidney transplantation: does socioeconomic status play a role? Am J Transplant 2012;12(2):369-78

48. Axelrod DA, Dzebisashvili N, Schnitzler MA, Salvalaggio PR, Segev DL, Gentry SE, Tuttle-Newhall J, Lentine KL. The interplay of socioeconomic status, distance to center, and interdonor service area travel on kidney transplant access and outcomes. Clin J Am Soc Nephrol. 2010;5(12):2276-88. 